\title{
1 Was ist Wahn?
}

\author{
Paul Hoff
}

Es fällt um einiges leichter zu beschreiben, was Wahn nicht ist, als zu definieren, was er ist. Und weil dies so ist, nähern sich, wie im vorliegenden Band, ganz unterschiedliche theoretische und methodische Ansätze dem gleichen Thema. In diesem ersten Beitrag werden zwei Perspektiven herausgearbeitet, die als Rahmen für die weitere Debatte um die Wahnfrage besonders geeignet erscheinen und die zu zwei Thesen verdichten werden sollen:

- Wahn ist in erster Linie ein psychopathologisches Phänomen.

- Wahn berührt im klinischen wie im Forschungskontext alle Grundsatzfragen der Psychiatrie. Dadurch wird er eo ipso zur Herausforderung für jede theoretische Richtung, die sich mit psychiatrischen und psychopathologischen Fragen befasst.

\subsection{Wahn als psychopathologisches Phänomen}

„Der Wahn galt durch alle Zeiten als das Grundphänomen der Verrücktheit, wahnsinnig und geisteskrank als dasselbe. Was der Wahn sei, ist in der Tat eine Grundfrage der Psychopathologie." (Jaspers 1946, S. 78 )

Diese Formulierung aus der 4. Auflage von Karl Jaspers’ „Allgemeiner Psychopathologie“ hat nichts an Aktualität eingebüßt, sieht man einmal von der sprachlichen Ausgestaltung ab. Denn wir treffen in allen Bereichen der Psychiatrie auf Wahnphänomene: 
- Bei den psychotischen Erkrankungen sind sie häufig dominierend, ja namengebend wie etwa bei der paranoiden Schizophrenie oder der anhaltenden wahnhaften Störung, früher Paranoia genannt.

- Doch finden wir Wahn auch bei affektiven Erkrankungen, vor allem bei schweren Verlaufsformen depressiver und manischer Zustände,

- wir beobachten Wahnphänomene bei organisch begründbaren psychischen Störungen, speziell bei Demenzen,

- im Kontext von substanzinduzierten Störungen im weitesten Sinne,

- ja selbst, wenn auch vergleichsweise selten, bei Persönlichkeitsstörungen.

Die pragmatische Umgrenzung von Wahn, wie sie in den meisten Kliniken und Praxen angewandt wird, kann sich wiederum auf Jaspers' Ausführungen berufen. Für diese Zwecke sind seine Umschreibungen sehr nützlich, jedoch - und Jaspers selbst war dies sehr bewusst - beginnen die Schwierigkeiten, wenn man jedes einzelne der jetzt zu nennenden Kriterien unter die Lupe nimmt.

\begin{abstract}
Üblicherweise wird Wahn im Jaspers'schen Sinne verstanden als Fehlbeurteilung der Realität, die subjektiv unmittelbar evident ist, als unabhängig von konkreter Erfahrung zutreffend erlebt wird („apriorische Evidenz“) und die mit einem Grad von Sicherheit, ja Unkorrigierbarkeit vertreten wird, auch und gerade im Angesicht aussagekräftiger Gegenargumente, die wir außerhalb von Wahnphänomenen nicht antreffen. Personen mit einem dergestalt definierten paranoiden Syndrom nehmen typischerweise erhebliche Nachteile in Kauf, da der wahnhafte Inhalt zum einen häufig ihre Lebensführung markant negativ beeinflusst, etwa durch soziale Isolierung, und zum anderen von der persönlichen Umgebung in aller Regel nicht geteilt, sondern vehement abgelehnt, ja für „verrückt“ erklärt wird.
\end{abstract}

Wer praktisch psychiatrisch arbeitet, weiß, dass es neben den idealtypischen paranoiden Zustandsbildern, bei denen alle genannten Kriterien erfüllt sind, eine viel größere Zahl von Übergangs- und Verdünnungsformen gibt, die aber gleichwohl zu erheblichem Leid und dringender Behandlungsbedürftigkeit führen können. Wenig Kontroversen wird es geben im Falle eines Patienten, der seit Jahren einen schweren Verfolgungswahn berichtet, der sich völlig zurückzieht, seine Wohnung kaum noch verlässt und seine gesamte Lebensführung den paranoiden Inhalten angepasst hat. Wie aber ist die Lage, wenn ein 40-jähriger, immer als zurückhaltend, vorsichtig, eigenbrötlerisch bekannter Versicherungskaufmann im Rahmen eines mehrmonatigen Konfliktes am Arbeitsplatz noch stiller wird, zusätzlich mürrisch, auch depressiv und sehr misstrauisch? Was bedeutet es, wenn er schließlich in einer Mischung aus Ärger und Ängstlichkeit äußert, man möge ihn ja ohnehin nicht, man schikaniere ihn, vielleicht beschatte und verfolge man ihn sogar, ja wolle ihn vergiften?

Und was ist in unserem Zusammenhang zu halten von einer ausgeprägt abergläubischen Person, die aus allerlei alltäglichen Situationen eine konkrete Bedeutung für sich selbst herausliest und damit vielleicht von Anderen als unsinnig oder riskant eingeschätzte Verhaltensweisen begründet? Wie fassen wir den psychischen Zustand einer Person, die sich, ohne früher jemals stärkere Auffälligkeiten gezeigt zu haben, ab einem bestimmten Zeitpunkt in ihrem Leben einer einzigen Idee verschreibt, sich in sie „verrennt“, sie zunehmend verteidigt und nachhaltig versucht, andere Menschen ebenfalls davon zu überzeugen? 
Betrachten wir diese in der Praxis häufigen Grenzfälle, so gelangen wir mit den gängigen Wahnkriterien rasch an eine Grenze. So etwa kann sich die unmittelbare und für die betroffene Person geradezu zwingende Evidenz des Wahninhaltes über zeitlich ausgedehnte Vorstadien entwickeln, in denen es sehr wohl zu Zweifeln und Alternativüberlegungen des Betroffenen kommt, Zweifeln allerdings, die ab einem bestimmten Punkt von der dann eintretenden Wahngewissheit nachhaltig beiseite geschoben werden. Die Lebensführung eines paranoiden Menschen muss auch nicht unbedingt markant beeinträchtigt werden. Mitunter gelingt es ihm oder ihr, Wahninhalte so zu verbergen und deren Auswirkungen im Alltag so wenig kenntlich zu machen, dass nicht einmal nahe stehende Personen davon wissen. Und schließlich gibt es die zunächst anachronistisch wirkenden Fälle, bei denen ein Wahninhalt de facto keine Fehlbeurteilung der Realität ist - man denke an einen lange und schwer alkoholabhängigen Mann, der einen klinisch eindeutigen Eifersuchtswahn entwickelt, wobei seine Frau sich allerdings tatsächlich von ihm abgewandt hat und heimlich eine neue Beziehung eingegangen ist.

Damit soll verdeutlicht werden, dass ein derart komplexes, die ganze Person und viele ihrer Interaktionen betreffendes Phänomen wie der Wahn nie auf eine einzige Erkenntnisebene - neurobiologisch, psychologisch, sozialwissenschaftlich - zu reduzieren und auch nicht durch wenige operationale Kriterien vollständig zu erfassen, geschweige denn zu definieren ist. Es bedarf immer der Einbettung in den gesamten psychopathologischen, biologischen und sozialen Kontext. Wahn ist insoweit eben nicht nur eine falsche Aussage, an der unbeirrbar festgehalten wird. Er ist auch nicht nur ein affektiver Zustand, etwa derjenige einer besonderen Verbissenheit und hartnäckigen Gereiztheit, nicht nur eine grundsätzliche „Verrückung“ der personalen Situation des Individuums in der Welt, etwa im Sinne des „Verlustes der natürlichen Selbstverständlichkeit“ (Blankenburg 1971, 1992) - er ist von alledem etwas, und dies wiederum bei jedem betroffenem Menschen in unterschiedlicher Graduierung.

Ungeachtet der ihm je unterlegten pathogenetischen Prämissen ist Wahn ein primär psychopathologisches Phänomen, das sich auf alle Lebensbereiche auswirken kann und das notwendig einen interaktionellen Aspekt hat, denn Wahn wird in der Regel, auf die eine oder andere Art, mitgeteilt. Freilich ist er kein isoliertes, gut abgrenzbares Einzelsymptom, sondern, ganz im Gegenteil, ein hochgradig komplexes, sich nur einer entsprechend differenzierten Betrachtung erschließendes Zustandsbild.

\subsection{Wahn als Schnittstelle psychiatrischer Grundsatzfragen}

Um plausibel zu machen, warum die in diesem Band beleuchteten Perspektiven zum Phänomen Wahn nicht zufällig so heterogen sind, sollen eine Reihe von grundsätzlichen Fragen der Psychiatrie angesprochen werden, die jeweils auch mit der Wahnproblematik eng zusammenhängen. Die These ist, dass Wahn eben nicht nur ein psychopathologisches Symptom unter vielen ist, sondern ein zentrales, mit jedem Verständnis von psychischer Störung, ja von Psyche schlechthin notwendig vernetztes Phänomen.

Es geht um die folgende Fragen und Bereiche:

- der Weg vom Symptom zur Krankheit

- Wahngenese und psychiatrischer Krankheitsbegriff 
- Psychopathologische Symptome: primäre oder sekundäre Phänomene?

- Psychopathologische Symptome: verständliche oder unverständliche Phänomene?

- Psychopathologische Symptome und die Grenze zwischen psychischer Gesundheit und Erkrankung

- Psychopathologische Symptome und ihr Einfluss auf Handlungssteuerung, Einsicht und Verantwortung der betroffenen Person

- Wie verhält sich die Psychopathologie zum Leib-Seele-Problem ${ }^{1}$ und zum Menschenbild?

\subsubsection{Der Weg vom Symptom zur Krankheit}

Schilderungen paranoiden Erlebens und Verhaltens durchziehen die psychiatrische Literatur seit Jahrhunderten. Niemals kontrovers war dabei die Einschätzung, dass es sich bei paranoiden Syndromen um besonders beeindruckende, oft auch befremdende und faszinierende Phänomene handelt. Sehr unterschiedlich hingegen haben Autoren die Frage beantwortet, ob es sich beim Wahn um einzelne Symptome handelt, um ein schon weit komplexeres, aus verschiedenen Einzelsymptomen und deren Interaktion zusammengesetztes Syndrom oder um eine eigentliche Krankheit. Und selbst bei letzterer Variante sind noch zwei Optionen zu unterscheiden: Sind Wahnerscheinungen immer Ausdruck einer anderen Erkrankung, etwa einer schizophrenen Psychose, oder kann Wahn, vor allem chronischer Wahn, auch als Krankheit sui generis auftreten, etwa im Sinne des Gaupp'schen Paranoiakonzeptes? Die pragmatischen operationalen Diagnosemanuale äußern sich zwar aus guten Gründen nicht direkt zu dieser Frage, stellen aber für den chronischen Wahn unter bestimmten Bedingungen eine eigene diagnostische Kategorie zur Verfügung (,anhaltende wahnhafte Störungen“, ICD-10 F22, „wahnhafte Störung“ DSM-IV 297.1).

\subsubsection{Wahngenese und psychiatrischer Krankheitsbegriff}

Bei aller unbestreitbaren Unübersichtlichkeit psychiatrischer Theorien von 1750 bis heute lassen sich doch - wie anderenorts umfassender ausgeführt (Hoff 2006) - einige grundsätzliche Positionen zur Genese psychischer Erkrankungen und damit auch des Wahnes unterscheiden:

1. Psychische Krankheit ist im Kern eine neurobiologische Dysfunktion, allenfalls sogar mit neuroanatomischem Substrat. Das Organ Gehirn steht im Mittelpunkt des Forschungs- und Therapieinteresses.

2. Psychische Krankheit ist im Kern eine biografisch verstehbare individuelle Fehlentwicklung. Es geht in erster Linie um hermeneutisches Arbeiten an Lebensgeschichten und -situationen.

3. Psychische Krankheit ist im Kern Ausdruck einer gesellschaftlich verankerten und letztlich nur dort verstehbaren, sich aber im Individuum manifestierenden Fehlentwicklung. Der Einfluss sozialer und kultureller Faktoren rückt wissenschaftlich wie klinisch in den Vordergrund.

1 Der Autor übernimmt hier aus praktischen Gründen den verbreiteten, wenn auch unscharfen Begriff „Leib-Seele-Problem“, freilich ohne damit einem unkritischen substantialistischen Seelenbegriff das Wort zu reden. 
4. Psychische Krankheit ist im Kern gerade keine „realwissenschaftlich“ erfassbare, insofern „echte“ Entität, die vollständig unabhängig vom Untersucher objektiv existiert und von diesem „erkannt“, „entdeckt“ werden muss. Sie kann nach aktuellem Wissensstand allenfalls, und zwar oft recht zuverlässig, mit operationalen Kriterien beschrieben, mitunter auch mit neuropsychologischen Parametern korreliert (aber eben im Unterschied zur Position 1 nicht erklärt) werden. Hier wird psychische Erkrankung in erster Linie zur deskriptiven Normabweichung. Die bekanntesten Beispiele für diesen Ansatz sind die heute international verbreiteten operationalen Diagnosemanuale ICD-10 (WHO 1991) und DSM-IV (APA 200o), die beide in der Weiterentwicklung zu den Nachfolgeversionen ICD-11 und DSM V stehen (Gaebel u. Zielasek 2008; Möller 2009).

Konkret bezogen auf Wahnphänomene führt uns das zu drei in der Tat differenten Herangehensweisen. Betrachten wir zunächst die neurobiologische Ebene. Eine konsistente neurobiologische Theorie zur Wahnentstehung liegt bislang nicht vor. Der Schwerpunkt der diesbezüglichen Forschung bezieht sich auf die Frage, ob sich bei paranoiden Menschen neurophysiologische und neuropsychologische Befunde von denen gesunder Personen, aber auch von denen nicht-paranoider psychisch erkrankter Personen unterscheiden. Auf der Ebene der Neurotransmitter im engeren Sinne gibt es keine eindeutigen Befunde. Bei evozierten Potenzialen wie der P3oo werden zwar Unterschiede zwischen schizophrenen und gesunden Personen berichtet, doch bleibt die Frage offen, welche Rolle dabei das Vorhandensein oder Fehlen paranoider Symptome spielt (Kunert et al. 2003, 2007).

Die Frage der Einbettung der Wahngenese in den jeweiligen Lebenslauf ist ein ebenso zentrales wie kontroverses Thema. Besondere Bekanntheit erreichte hier der Fall des „Hauptlehrers Wagner“, eines paranoiden, möglicherweise schizophrenen Patienten des Tübinger Psychiaters Robert Gaupp (1870-1953), der im Jahre 1906 in einem psychotischem Zustand mehrere gravierende Gewaltdelikte verübte und anschließend wegen seiner chronischen Wahnerkrankung für Jahrzehnte in psychiatrischer Untersuchung und Behandlung verblieb (Gaupp 1920; Neuzner u. Brandstätter 1996). Nicht zuletzt vor dem Hintergrund dieses Falles bekräftigte Gaupp - allerdings inhaltlich markant anders als psychoanalytische Autoren (Freud 1911/1978) - die Auffassung, dass chronischer Wahn unter bestimmten Umständen sehr wohl und in hohem Maße psychologisch ableitbar, ,verständlich“ sei, auch wenn dies auf den ersten Blick nicht so erscheine. Die Frage der Verständlichkeit oder Unverständlichkeit von psychiatrischen Symptomen wird noch zur Sprache kommen. An dieser Stelle kommt es auf den Anspruch von Gaupps Ansatz an, durch die psychologischen und psychodynamischen Zusammenhänge nicht nur den Verstehenshorizont für eine gravierende psychische Erkrankung zu erweitern, sondern auch zu deren Ätiologie Wesentliches beizutragen. Letzteres setzte aber für Gaupp - und noch deutlicher für seinen Schüler Ernst Kretschmer (1888-1964) (Kretschmer 1918) - eine bestimmte Matrix von psychologischen und sozialen Bedingungen voraus, vor allem eine sensitive Persönlichkeit in Verbindung mit wiederholten kränkenden oder beschämenden Lebensereignissen.

In jüngerer Zeit hat sich ein psychologischer Befund bei paranoiden Patienten als recht robust herausgestellt, der zwar weniger auf die Inhalte und die Lebenssituationen abhebt, wohl aber auf den formalen Aspekt, wie viele Informationen eine Person benötigt, um zu einer für sie als sicher erlebten Aussage zu gelangen (Bottlender et al. 1999; Garety 1991; Garety et al. 2007; Moritz u. Woodward 2005). Dieser Ansatz des 
„probabilistic reasoning“ konnte belegen, dass paranoide Personen meist signifikant weniger Informationen über eine bestimmte Situation anfordern, um sie sicher einschätzen zu können, als dies bei psychisch unauffälligen Personen oder, nicht verwunderlich, bei depressiven Menschen der Fall ist, die sehr viel mehr auf Information und Rückversicherung angewiesen sind, um sich überhaupt entscheiden zu können. Sowohl die empirische Bearbeitung als auch die konzeptuelle Weiterentwicklung neuropsychologisch orientierter Ansätze in der Wahnforschung haben in den vergangenen Jahren an Dynamik gewonnen (Bentall 1994; Bentall et al. 2001; Blackwood et al. 2001; Freeman et al. 2002; Frith u. Frith 1999).

Eine psychopathologisch differenzierte deskriptive Herangehensweise an Wahn haben etwa Karl Jaspers und Kurt Schneider vorbereitet und vertreten. Sie wird in den heutigen operationalen Diagnosemanualen ICD-1o und DSM-IV konsequent fortgesetzt. Freilich war und blieb für Jaspers bei aller Deskriptivität, die das Phänomen präzise $\mathrm{zu}$ erfassen und vorschnelle Interpretationen zu meiden habe, der zentrale Bezugspunkt das Erleben des Patienten. Er sprach, allerdings nicht nur in diesem Zusammenhang, von der „phänomenologischen Methode“ in der Psychiatrie (Jaspers 1912). Alle psychischen Vorgänge, ob gesund oder krank, also auch der Wahn, waren für ihn niemals unmittelbar beobachtbar, sondern nur erschließbar über Ausdruck und Äußerungen des Erlebenden, seien diese sprachlicher, psychomotorischer, behavioraler oder auch gestaltend-künstlerischer Natur.

Jaspers kam es wesentlich auf die Unterscheidung zwischen „echtem“ Wahn und bloß „wahnähnlichem“ Erleben an. Für ihn entsprach das Auftreten von echtem Wahn einem qualitativen Sprung, etwas völlig Neues trete hier auf. Jaspers hielt einen solchen echten Wahn für psychologisch unableitbar und damit unverständlich, zumindest in seinem Kern.

Für Kurt Schneider hatte der deskriptive Zugang neben der psychopathologischen Differenzierung vor allem für klare Begriffe zu sorgen. Freilich sollten diese - genau wie bei Jaspers - das Psychische gerade nicht in unverbunden nebeneinander stehende Einzelelemente aufsplittern, sondern, wo dies möglich ist, in kritisch reflektierter Weise den verstehenden Gesamtzusammenhang wahren. Hinsichtlich des Wahnes widmete sich Schneider verstärkt dessen formaler Struktur: Er suchte nach einem Kriterium, welches zuverlässig zwischen wirklichem Wahn und Phänomenen, die ihm nur ähneln, zu unterscheiden vermöge. Dies führte ihn zur Beschreibung der logisch zweigliedrigen Wahnwahrnehmung: Die ungestörte Sinneswahrnehmung werde in einem zweiten, dem eigentlich krankhaft veränderten Schritt, wahnhaft fehlgedeutet. Beispielsweise bedeute das Erblicken einer dunklen Gewitterwolke, dass die wahrnehmende Person bald sterben werde. Hier liege echter Wahn vor (Schneider 1980).

Es wird deutlich, wie sehr es auch bei einem konsequent deskriptiven Vorgehen nicht bei der strengen Beschreibung bleibt, ja bleiben kann. Psycho(patho)logische Phänomene sind niemals isoliert zu sehen, gleichsam atomistisch. Sie sind immer miteinander vernetzt und sagen für die betreffende Person etwas aus, bedeuten etwas. Dies gilt für „einfache“ psychopathologische Symptome wie eine Denkbeschleunigung oder eine phobische Angst ebenso wie für besonders komplexe Phänomene wie Wahn oder Störungen des Ich-Erlebens. 


\subsubsection{Psychopathologische Symptome: primäre oder sekundäre Phänomene?}

Eine grundlegende Frage ist, ob ein bestimmtes psychopathologisches Phänomen genuin als solches entsteht, quasi aus sich heraus, oder eben Folge eines anderen, eigentlich krankhaften Zustandes ist. Und so diskutiert die wissenschaftliche Wahnliteratur regelmäßig, wie es denn sein könne, dass eine ansonsten rational agierende, affektiv und kognitiv kompetente Person plötzliche beginne, sehr unwahrscheinliche, ja geradezu unmögliche Sachverhalte mit einem ungekannten Grad an subjektiver Sicherheit zu behaupten und dadurch markante persönliche Nachteile in Kauf zu nehmen.

Im 19. Jahrhundert standen sich im Rahmen der damals intensiv geführten Debatte um die Einheitspsychose zwei Lager gegenüber: Für die einen war es durchaus möglich, dass Wahn gleichsam aus dem Nichts entstehe, als primäres Symptom, das auftauche, ohne dass im Vorfeld sonstige Störungen zu beobachten gewesen wären. Snell (1865) sprach in diesem Sinne vom „primären Irresein“. Demgegenüber vertrat Wilhelm Griesinger (1817-1868), aufbauend auf Argumenten seines Lehrers Albrecht Zeller (1804-1877), die These, es gebe in der Psychiatrie nicht eine Vielfalt von Psychosen, sondern nur gerade eine, eben die „Einheitspsychose“. Diese verlaufe allerdings in Stadien, ein Prozess, der bei einem Patienten früh, etwa bei einem milden affektiven Bild, stoppen, bei einem zweiten hingegen die gesamte Bandbreite bis hin zur schweren Demenz durchlaufen könne. Wahn war für ihn dabei zunächst einmal etwas zeitlich Nachgeordnetes, also Sekundäres: Jede schwere psychische Erkrankung beginnt nach dieser Auffassung mit einem affektiven Stadium. Dieses könne im günstigsten Falle ausheilen, könne sich aber auch zu einem paranoiden und katatonen Zustandsbild entwickeln oder gar, wie erwähnt, zu einem weitgehenden Verlust kognitiver Fähigkeiten führen (Griesinger 1861; Mundt u. Sass 1992). Betont werden sollte, dass sich Griesinger in späteren Veröffentlichungen der Snellschen Position angenähert und zumindest die Möglichkeit primärer paranoider Symptome anerkannt hat. Für uns heute mag diese Debatte vor allem deswegen interessant sein, weil sie die Bedeutung der Affektivität für die Entstehung und Aufrechterhaltung von Wahn unterstreicht, auch wenn wohl nicht viele so weit gehen würden wie Specht (1905), für den - freilich schon vor mehr als 100 Jahren - die Paranoia eine besondere Verlaufsform der chronischen Manie darstellte.

Natürlich kann sich die Frage nach der Ableitbarkeit psychischer und psychopathologischer Phänomene auch auf die Frage ihrer (als kausal gedachten) Rückführbarkeit auf somatische Vorgänge beziehen. Konkreter auf unser Thema bezogen, hieße das: Kommt Wahn nur beim Vorliegen bestimmter neurobiologischer Ursachenbündel vor oder kann er sich völlig unabhängig von diesen entwickeln?

\subsubsection{Psychopathologische Symptome: verständlich oder unverständlich?}

Auch hier treffen wir wieder auf eine grundsätzliche Frage, die eben weit mehr ist als ein Detailproblem der psychiatrischen Diagnostik: Haben psychopathologische Symptome eine wesentliche individuelle Bedeutung, einen „Sinn“ im Lebenskontext der betreffenden Person oder sind sie schlicht Indikatoren einer „zugrunde liegenden" Krankheit, etwa wie Bojen in einem Fluss, die der Schifffahrt Untiefen anzeigen. Eine differenzierte Darstellung dieser epistemologischen Aspekte des Symptombegriffes findet sich bei Berrios (1995) sowie Markova und Berrios (2009). 
Wiederum ist der Wahn ein besonders gutes Exempel: Es überschneiden sich zwei dichotome Argumentationsstränge, nämlich die auf die Grenze der Methode des Verstehens bezogene Abtrennung verständlicher und unverständlicher (Teil-)Inhalte des Wahnes einerseits und die ätiologische Abtrennung psychologisch und „organisch“ begründbarer Wahnphänomene andererseits. Eine aus der Heidelberger Tradition stammende Auffassung sieht mit Blick auf die Wahninhalte ein weites Feld für die Verstehbarkeit, auch bei solchen Inhalten, die auf den ersten Blick befremdlich, ja bizarr anmuten mögen. Freilich gelinge dies nur bei subtiler Kenntnis von Lebensgeschichte und Persönlichkeit der betroffenen Person. Hingegen stehe man bei dem Faktum, dass es überhaupt einen Wahn gebe, egal welchen Inhaltes, vor etwas Unverständlichem. So ist für Karl Jaspers das primäre Wahnerlebnis grundsätzlich nicht psychologisch ableitbar. Ein solcher primärer Vorgang gehe überdies mit einer „Umwandlung der Persönlichkeit“ einher, was schließlich in die völlige Unkorrigierbarkeit bei ansonsten ungestörten kognitiven Abläufen münde. Das primäre Wahnerlebnis sensu Jaspers muss keine konkrete Idee sein, sondern zum Beispiel ein vages Erleben, bei dem der Patient betont, irgendetwas stimme ernsthaft nicht, bedrohe ihn, er wisse aber weder was noch warum. So kommt Jaspers zu einer Unterscheidung echter Wahnideen von bloß wahnhaften Ideen, ein Grenze allerdings, die sich sowohl in klinischer wie theoretischer Perspektive als keineswegs hermetisch herausgestellt hat:

„Nur diejenigen Wahnideen, die auf ein primäres pathologisches Erleben als Quelle zurückweisen oder zu ihrer Erklärung eine Umwandlung der Persönlichkeit als Voraussetzung fordern, nennen wir echte Wahnideen. [...] Diejenigen Wahnideen dagegen, die uns verständlich aus anderen seelischen Vorgängen hervorgegangen sind, die wir also psychologisch zurückverfolgen können auf Affekte, auf Triebe, Wünsche und Befürchtungen, zu deren Erklärung wir keine Umwandlung der Persönlichkeit brauchen, [...] nennen wir wahnhafte Ideen." (Jaspers 1946, S. 89)

Hans Gruhle, wie Jaspers dem Heidelberger Kreis zugehörig, geht noch einen Schritt weiter und spricht die ätiologische Ebene explizit an:
„Das Interesse gilt nicht in erster Linie der Frage nach dem speziellen Inhalt der Wahnidee..., nicht einmal der Frage, warum jemand überhaupt verfolgt wird, sondern warum jemand überhaupt Wahnideen hat. Ich halte den echten Wahn für ein Primärsymptom der Schizo- phrenie, für ein unableitbares, unverständliches, organisches Symptom." [Hervorhebungen von Gruhle] (Gruhle 1932, S. 178)

Die für die Psychiatrie so bedeutsame methodische Trias von Erklären, Verstehen und Beschreiben (Hoff 2010) kommt auch im Bereich des Wahnes zur Geltung. Dabei handelt es sich um unterscheidbare Modi des wissenschaftlichen Erfassens psychischer Phänomene. Diese Modi haben immer Überschneidungsbereiche, kommen also in „reiner“ Form nicht vor. Insoweit sind sie keine kategorialen, einander ausschließenden Gegensätze, sondern sich wechselseitig ergänzende Perspektiven.

\subsubsection{Psychopathologische Symptome und die Grenze zwischen psychischer Gesundheit und Krankheit}

Symptome, die eine behandlungsbedürftige Erkrankung anzeigen, von ungestörten oder nur leicht auffälligen psycho(patho)logischen Phänomenen abzugrenzen, kann ausgesprochen schwierig sein. Man denke nur an die große Bandbreite der Begriffe „traurig“ oder „ängstlich“. Im Falle des Wahnes präsentiert sich dieses generelle Pro- 
blem in zugespitzter Form: Zum einen ist es kaum möglich, ohne umfassende Kenntnis der betreffenden Person in seriöser Weise zu differenzieren zwischen situationsadäquatem Misstrauen, paranoider Haltung und manifestem Wahn. Dies gilt erst recht, wenn, wie so oft, ein zeitlich fluktuierendes und inhaltlich sehr dynamisches Zustandsbild vorliegt. Zum anderen - das wesentlich heiklere Thema - geraten wir bei allen Versuchen, Wahn begrifflich zu fassen, in das Dilemma, ihn abzugrenzen von fest gefügten, eng mit der Persönlichkeit verbundenen, ja in ihr verankerten und daher sehr stabilen Grundüberzeugungen einer Person - notabene: sei diese nun psychisch gesund oder nicht. Hier sind zu nennen Werthaltungen, Weltanschauungen, politische Überzeugungen und die religiöse Orientierung. All das hat mit Psychopathologie zunächst wenig zu tun. Das ändert sich, wenn sich Inhalt oder Form einer persönlichen Überzeugung in eine extreme Richtung bewegen, dann nämlich kommen wir in den vielgestaltigen Bereich von überwertiger Idee, Starrsinn, Fanatismus und Querulanz. Die Frage, ob und inwieweit sich der Psychopathologe zu diesen Themen äußern soll, muss mit Zurückhaltung und großer Sorgfalt, aber ohne falsche Scheu beantwortet werden.

Zwei Aspekte scheinen mir wesentlich: Weltanschauliche und religiöse Überzeugungen entstehen typischerweise über lange Zeiträume, sie wachsen quasi mit der Person. Grundsätzlich verfügen Menschen über die Fähigkeit, eigene Grundhaltungen kritisch zu hinterfragen. Diese Fähigkeit zu entwickeln und zu fördern, ist eine zentrale Aufgabe der Erziehung. Natürlich fällt die fundamentale Infragestellung der eigenen Person sehr viel schwerer als das Umgehen mit (Selbst-)Kritik in weniger bedeutsamem, alltäglichem Kontext. Aber auch persönliche Einstellungen - aus Sicht des strukturdynamischen Ansatzes des Heidelberger Psychopathologen Werner Janzarik zentrale Bestandteile seelischer Struktur (Janzarik 1988) - sind nicht völlig starr, nicht unveränderbar, nicht „unkorrigierbar“. Im klinischen Kernbereich von Wahn hingegen treffen wir genau auf diese sehr spezifische Art von Unkorrigierbarkeit, die in jüngeren Arbeiten zum Wahn als wesentliches Element des pathologischen Ceschehens verstanden wird (Spitzer 1989, 1995).

Auch bei den zahlreichen Spielarten des Fanatismus liegt zumeist eine längere Wegstrecke hinter der betreffenden Person, in der sich die Einstellung zunehmend verfestigte, radikalisierte und durch Einflüsse Gleichgesinnter zusätzliche Unterstützung erfuhr. Bei solchen biografischen Entwicklungen, die zu einer extremen Einengung von Verhaltensweisen und Lebenszielen führen können, sind Übergänge in manifest paranoide Syndrome denkbar (und vor allem dem forensisch tätigen Psychiater gut bekannt). Ein klassisches Beispiel sind querulatorische Fehlentwicklungen, die in einen Querulantenwahn münden.

Eines aber kann nicht deutlich genug hervorgehoben werden: Das Eintreten für eine persönlich als richtig und wertvoll betrachtete Zielsetzung auch gegen ausgeprägte oder längere Widerstände hat eo ipso nichts mit psychischer Erkrankung zu tun, auch nicht mit Wahn. Es ist Aufgabe der Psychiatrie, zu verhindern, dass ihre Fachtermini, insbesondere psychopathologische und diagnostische, missbräuchlich verwendet werden - im schlimmsten Fall zur systematischen Pathologisierung abweichender Meinungen. Beispiele, die zeigen, dass dies eine nur zu begründete Warnung ist, finden sich in der Ceschichte unseres Faches genügend. In summa: Einzelne psychopathologische Symptome sind, unabhängig von ihrem Ausprägungsgrad, nicht geeignet, das Vorliegen einer spezifischen psychischen Erkrankung, auch nicht einer wahnhaften Störung, zu begründen. 
Eine psychische Störung, insbesondere auch eine paranoide Störung, kann niemals nur auf einem gemäß statistischer Norm abweichenden Verhalten oder auf dazugehörigen Äußerungen begründet werden.

\subsubsection{Psychopathologische Symptome und ihr Einfluss auf Handlungssteuerung, Einsicht und Verantwortung der betroffenen Person}

Mitunter verwenden Psychiater den Begriff „Krankheitseinsicht“ mit einer gewissen Selbstverständlichkeit, so als sei er ganz unproblematisch. Das ist er nicht. Ein ambulanter Patient mit einer episodisch verlaufenden schizophrenen Erkrankung, bekannt für seine spitze Zunge, machte sich einmal über diesen Begriff lustig, indem er ihn so definierte: „Krankheitseinsicht liegt dann und nur dann vor, wenn der Patient der selben Auffassung ist wie der Behandler." Zwar pointiert, doch im Grunde zutreffend erfasst diese Formulierung die Grundfrage, von welchem Standpunkt aus pathologisches Erleben und Verhalten beurteilt wird und wie die verschiedenen Perspektiven sinnvoll, sprich: therapieorientiert, integriert werden können. Wird für den Betroffenen das vom Experten als pathologisch bezeichnete Erleben als pathologisch wahrnehmbar? Inwiefern wird seine Fähigkeit zum Monitoring des eigenen Erlebens, zur Selbstkritik etwa durch eine schwere depressive Hemmung, ein paranoides Misstrauen oder eine ausgeprägte Ich-Störung eingeschränkt oder aufgehoben?

Nun wird im Falle eines manifesten Wahnsystems am Versagen bestimmter Korrektur- und Kritikmechanismen kaum gezweifelt werden können, denn gerade darin besteht ein zentrales Charakteristikum des Wahns. Doch schließt dies keineswegs aus, sich in der psychotherapeutischen Arbeit dem heiklen Thema zu nähern. So könnte der Therapeut dem paranoiden Patienten gegenüber zu begründen versuchen, warum er von einer psychischen Störung ausgeht und vor allem warum diese Störung die Lebensqualität markant beeinträchtigt. Selbst wenn dadurch die Kernpunkte des Wahnes untangiert bleiben sollten - was sie häufig tun -, so kann sich doch ein tragfähiges therapeutisches Bündnis entwickeln. Der psychopathologische Befund - das ist hier der Kernpunkt - arbeitet grundsätzlich nicht mit unbezweifelbaren objektiven Messwerten, sondern ist das Ergebnis eines notwendig interaktiven Vorganges zwischen Patient und Untersucher.

Diese klinischen Überlegungen gelten grundsätzlich auch für die forensische Situation. Hier kommt als spezielle Problematik hinzu, dass der oder die Sachverständige sich zu einem allfälligen Zusammenhang zu äußern hat zwischen paranoider Symptomatik einerseits und der Fähigkeit zur Einsicht in das Verbotene einer bestimmten Handlung und zur angemessenen Handlungssteuerung andererseits. Wahn allein kann hier als psychopathologisches Kriterium nicht ausreichen. So mag ein chronisch schizophrener Patient deutlich wahnhaft, aber sehr wohl in der Lage sein einzusehen, dass ein Ladendiebstahl eine strafbare Handlung ist. Greift er hingegen eine fremde Person aus wahnhafter Situationsverkennung heraus körperlich an, so wird unter Berücksichtigung des gesamten psychopathologischen Befundes wahrscheinlich eine forensisch maßgebliche Beeinträchtigung erwogen werden. Ob diese nun der Einsichts- oder, wie es die juristische Praxis bevorzugt, der Steuerungsfähigkeit zugerechnet wird, ist ein kontroverses, hier nicht weiter zu erörterndes Thema (Janzarik 1991, 2000). 


\subsubsection{Wie verhält sich die Psychopathologie zum Leib-Seele-Problem² und zum Menschenbild?}

Schließlich sei - wieder unter besonderer Berücksichtigung des Wahnes - daran erinnert, dass es sich bei der Psychopathologie um ein auf sehr verschiedenen Ebenen operierendes wissenschaftliches und praktisch-klinisches Gebiet handelt. In jüngerer Zeit ist freilich die Psychopathologie in mancherlei Hinsicht in die Defensive geraten (Hoff 2009). Doch könnte sie zukünftig dann wieder eine tragende Rolle in der und für die Psychiatrie spielen, wenn sie jenseits ihres Status als Befunderhebungstechnik zu einer inhaltlichen und methodenkritischen Klammer für das gesamte Fach wird, die die konkurrierenden psychiatrischen Konzepte miteinander ins Gespräch bringt und so zu einer Identitätsbildung des Faches beiträgt.

Die Psychopathologie hat oft wesentliche Funktionen für die Psychiatrie übernommen, auch wenn sie dies oft nicht klar genug reflektiert und geäußert hat: Sie hat eine deskriptive Ebene, die der Kommunikation von Untersuchern, Behandlern und Forschern untereinander dient. Sie verfügt über eine klinische Ebene, die Diagnosen und andere klassifikatorische Ansätze unterstützt oder ermöglicht. Und schließlich beinhaltet sie eine strukturale Ebene, bei der es um das wissenschaftliche Erfassen von motivationalen Zusammenhängen und komplexen Bedeutungen psychischer Sachverhalte geht, etwa mit Blick auf Lebensläufe, Persönlichkeitseigenschaften oder die Bewertung komplexer Handlungsabläufe wie in der forensischen Begutachtung - generell also Phänomenbereiche, die durch eine einfache kriteriengeleitete Deskription nicht hinreichend erfasst werden können (Hoff 2008; Stanghellini 2009).

Diese unbestrittenen und vorwiegend klinisch orientierten Ebenen der Psychopathologie wären künftig wieder verstärkt zu ergänzen durch zwei weitere: Zum Einen die Ebene der Methodenkritik, wo die Frage im Vordergrund steht, welchen Gültigkeitsbereich Aussagen haben, die auf einer einzelnen wissenschaftlichen Methode (oder einer Kombination mehrerer Methoden) beruhen. Beispiele wären die Aussagenbereiche der deskriptiven (in der Regel kriteriengeleiteten) klinischen Diagnostik, der zerebralen Bildgebung mittels fMRI, der neuropsychologischen Leistungsdiagnostik oder der - meist im Bereich der Ergotherapie angesiedelten - Arbeitsdiagnostik.

In einem weiteren, noch komplexeren Schritt hätte die Psychopathologie zukünftig wieder vermehrt die Aufgabe, die allgemeinen wissenschaftstheoretischen bzw. philosophischen Fragen in die psychiatrische Debatte in Klinik und Forschung einzubringen. Gemeint sind Fragen wie die nach der Relation neurowissenschaftlicher, psychologischer und sozialwissenschaftlicher Befunde, nach der gesellschaftlichen und kulturellen Bedingtheit psychiatrischer Konzepte und schließlich - der heikelste Punkt nach dem Menschenbild, das die Psychiaterin und der Psychiater ihrem täglichen Handeln unterlegen - und sei es noch so implizit.

Das folgende Schema (s. Abb. 1) illustriert dieses erweiterte Verständnis von Psychopathologie:

2 vgl. Fußnote 1 


\section{Psychopathologie als Klammer}

\begin{tabular}{|l|l|}
\hline - & Deskriptiv: Kommunikation \\
- Stinisch: Diagnose und Klassifikation \\
Bedeutungen
\end{tabular}

Abb. 1 Ein erweitertes Verständnis von Psychopathologie, mod. nach Stanghellini (2009) und Hoff (2008)

Konkret heißt dies, dass die Psychopathologie einerseits ihre klinischen Begriffe auf der operationalen wie auf der qualitativen Ebene kontinuierlich weiterentwickelt und aktiv eine sinnvolle inhaltliche Vernetzung mit den Neuro- und Sozialwissenschaften betreibt. Neben diesen praktischen Aspekten hätte sie aber auch auf der theoretischen Ebene dafür zu sorgen, dass Subjektivität und personale Autonomie als Gegenstände von Wissenschaft anerkannt und nicht durch vermeintlich selbsterklärende empirische Befunde „eliminiert“ werden, seien diese Befunde nun neurobiologischer, neuropsychologischer oder sozialwissenschaftlicher Art. Das kritische Bewusstsein um die Grenzen der Aussagekraft der jeweiligen Methode muss überdies nachhaltig in der psychiatrischen Ideengeschichte verankert sein.

\section{Resümee}

Die Titelfrage ist nicht mit einer Aussage zu beantworten. Doch sollten zwei Aspekte verdeutlicht werden, bevor sich die weiteren Kapitel des vorliegenden Buches detaillierter mit den verschiedenen einzelnen Perspektiven befassen:

- Wahn hat als psychopathologischer Phänomenbereich eine außerordentlich komplexe Binnenstruktur, ist also sicher kein isolierter oder gar einfacher Sachverhalt.

- Jede vertiefte Beschäftigung mit Wahn - unabhängig von der angewandten Methode - führt notwendig über die klinisch-psychopathologische Ebene hinaus und bringt grundsätzliche Fragen des Fachgebietes ins Spiel.

Es gibt viele gute Gründe, skeptisch zu sein, wenn diese Grundfragen wie die Beziehung zwischen psychischen und körperlichen Phänomenen, der Stellenwert von Subjektivität und personaler Autonomie oder, ganz aktuell, die Bedeutung neurowissenschaftlicher Befunde für unser Menschenbild in apodiktischer Art diskutiert oder beantwortet werden. Es ist nicht zuletzt der Wahn, der sich jeder einseitig verkürzenden Erfassung hartnäckig verweigert. Dies aber ist bei einem so komplexen Phänomen gerade kein Nachteil, sondern Chance für eine methodisch reflektierte Forschung - auch insoweit ist Wahn eine pars pro toto für die Psychiatrie. 


\section{Literatur}

American Psychiatric Association (APA) (2000) Diagnostic and statistical manual of mental disorders (4th edition, text revision) (DSM-IV-TR). APA Washington DC

Bentall RP (1994) Cognitive biases and abnormal beliefs: towards a model of persecutory delusions. In: David AS, Cutting I (Hrsg.) The Neuropsychology of Schizophrenia. 337-360. Lawrence Erlbaum London

Bentall RP, Corcoran R, Howard R (2001) Persecutory delusions: a review and theoretical integration. Clin Psychol Rev 21, 1143-1192

Berrios GE (1995) The history of mental symptoms. Descriptive psychopathology since the 19th century. Cambridge University Press Cambridge

Blackwood N), Howard RJ, Bentall RP (2001) Cognitive neuropsychiatric models of persecutory delusions. Am I Psychiatry 2, 527-539

Blankenburg W (1971) Der Verlust der natürlichen Selbstverständlichkeit. Enke Stuttgart

Blankenburg W (1992) Analysen der Verselbstständigung eines Themas zum Wahn. In: Kaschka WP, Lungershausen E (Hrsg.) Paranoide Störungen. 17-32. Springer Berlin

Bottlender R, Buchberger A, Hoff P, Möller HI (1999) Urteilsbildung und Wahn. Eine Studie zum Urteilsverhalten von wahnhaften, depressiven und gesunden Probanden. Nervenarzt 70, 987-992

Freeman D, Garety PA, Kuipers E (2002) A cognitive model of persecutory delusions. Br I Clin Psychol 41, 331-347

Freud S (1978) Psychoanalytische Bemerkungen über einen autobiographisch beschriebenen Fall von Paranoia (dementia paranoides), 1911. 239-320. Gesammelte Werke VIII, Fischer Frankfurt am Main

Frith CD, Frith U (1999) Interacting minds - a biological basis. Science 286, 1692-1695

Gaebel W, Zielasek I (2008) The DSM-V initiative "deconstructing psychosis“" in the context of Kraepelin's concept on nosology. Eur Arch Psychiatry Clin Neurosci 258 (Suppl 2), 41-47

Garety PA (1991) Reasoning and delusion. Br | Psychiatry 159, 14-18

Garety PA, Bebbington P, Fowler D (2007) Implications for neurobiological research of cognitive models of psychosis. Psychol Med 37, 1377-1391

Gaupp R (1920) Der Fall Wagner. Eine Katamnese, zugleich ein Beitrag zur Lehre von der Paranoia. Z Ges Neurol Psychiatr 60, 312-327

Griesinger W (1861) Die Pathologie und Therapie der psychischen Krankheiten. 2., umgearbeitete und sehr vermehrte Auflage. Krabbe Stuttgart

Gruhle HW (1932) Die Psychopathologie. In: Bumke 0 (Hrsg.) Handbuch der Geisteskrankheiten. Spezieller Teil. Fünfter Teil. Die Schizophrenie. 135-210. Springer Berlin

Hoff P (2006) Leib \& Seele, Gehirn \& Geist, Gesundheit \& Krankheit: Die Psychiatrie als Schnittstelle medizinischer, philosophischer und gesellschaftlicher Kontroversen. In: Hermanni F, Buchheim T (Hrsg.) Das Leib-Seele-Problem. Antwortversuche aus medizinisch-naturwissenschaftlicher, philosophischer und theologischer Sicht. 39-67. Wilhelm Fink München

Hoff P (2008) Do social psychiatry and neurosciences need psychopathology - and if yes, what for? Int Rev Psychiatry 20, 515-520

Hoff P (2009) Historical roots of the concept of mental illness. In: Salloum IM, Mezzich JE (Hrsg.) Psychiatric diagnosis - challenges and prospects. 3-14. Wiley-Blackwell Chichester

Hoff P (2010) Psychische Störungen: erklären, verstehen oder beschreiben? Historische und aktuelle Perspektiven einer psychopathologischen Kernfrage. Schweizer Archiv für Neurologie und Psychiatrie 161, 200-208

Janzarik W (1988) Strukturdynamische Grundlagen der Psychiatrie. Enke Stuttgart

Janzarik W (1991) Grundlagen der Einsicht und das Verhältnis von Einsicht und Steuerung. Nervenarzt 62, 423-427

Janzarik W (2000) Handlungsanalyse und forensische Bewertung seelischer Devianz. Nervenarzt 71, 181-187

Jaspers K (1912) Die phänomenologische Forschungsrichtung in der Psychopathologie. Zschr ges Neurol Psychiat 9, 391-408

Jaspers K (1946) Allgemeine Psychopathologie, 4. Aufl. Springer Berlin Heidelberg New York

Kretschmer E (1918) Der sensitive Beziehungswahn. Ein Beitrag zur Paranoiafrage und zur psychiatrischen Charakterlehre. Springer Berlin 
Kunert HI, Prüter C, Hoff P (2003) Wahn - eine neurobiologische und neuropsychologische Bestandsaufnahme. In: Schiepek G (Hrsg.) Neurobiologie der Psychotherapie. 436-452. Schattauer Stuttgart New York

Kunert HJ, Norra C, Hoff P (2007) Theories of delusional disorders. An update and review. Psychopathology 40, 191-202

Markova IS, Berrios GE (2009) Epistemology of mental symptoms. Psychopathology 42, 343-349

Möller HJ (2009) Development of DSM-V and ICD-11: Tendencies and potential of new classifications in psychiatry at the current state of knowledge. Psychiatr Clin Neurosci 63, 595-612

Moritz S, Woodward TS (2005) Jumping to conclusions in delusional and not delusional schizophrenic patients. Br J Clin Psychol 44, 193-207

Mundt C, Sass H (Hrsg.) (1992) Für und Wider die Einheitspsychose. Thieme Stuttgart

Neuzner B, Brandstätter H (1996) Wagner. Lehrer, Dichter, Massenmörder. Eichborn Frankfurt am Main

Schneider K (1980) Klinische Psychopathologie. 12. Auflage. Thieme Stuttgart, New York

Snell L (1865) Über Monomanie als primäre Form der Seelenstörung. Allg Zschr Psychiat 22, 368-381

Specht G (1905) Chronische Manie und Paranoia. Centralblatt für Nervenheilkunde und Psychiatrie 28, 590-597

Spitzer M (1989) Was ist Wahn? Untersuchung zum Wahnproblem. Springer Berlin

Spitzer M (1995) A neurocomputational approach to delusions. Compr Psychiatry 36, 83-105

Stanghellini G (2009) The meanings of psychopathology. Curr Opin Psychiatry 22, 559-564

World Health Organisation (WHO) (1991) Tenth revision of the International Classification of Diseases, chapter V

(F): Mental and behavioural disorders (including disorders of psychological development). Clinical descriptions and diagnostic guidelines. WHO, Geneva [deutsch (1991): ICD-10. Huber, Bern Göttingen Toronto] 